\title{
Survival is affected by mutation type and molecular mechanism in vascular Ehlers-Danlos syndrome (EDS type IV)
}

\author{
Melanie G. Pepin, MS1, Ulrike Schwarze, MD¹, Kenneth M. Rice, PhD², Mingdong Liu, PhD², \\ Dru Leistritz, MS ${ }^{1}$ and Peter H. Byers, MD ${ }^{1,3}$
}

Purpose: We sought to characterize the natural history of vascular Ehlers-Danlos syndrome in individuals with heterozygous COL $3 A 1$ mutations.

Methods: We reviewed clinical records for details of vascular, bowel, and organ complications in 1,231 individuals (630 index cases and 601 relatives).

Results: Missense and splice-site mutations accounted for more than $90 \%$ of the 572 alterations that we had identified in COL3A1. Median survival was 51 years but was influenced by gender (lower in men) and by the type of mutation.
Conclusion: Although vascular Ehlers-Danlos syndrome appears to be genetically homogeneous, allelic heterogeneity is marked, and the natural history varies with gender and type of mutation in COL $3 A 1$. These findings indicate that when counseling families, confirmation of the presence of a COL $3 A 1$ mutation and its nature can help evaluate the risks of complications. These data are also important ingredients in both the selection and allocation of individuals to appropriate arms in clinical trials to assess the effects of interventions.

Genet Med advance online publication 12 June 2014

Key Words: Ehlers-Danlos syndrome type IV; missense mutations; nonsense mutations; splice-site mutations; survival

\section{INTRODUCTION}

The clinical diagnosis of vascular Ehlers-Danlos syndrome (vEDS; also known as EDS type IV) can be suspected on the basis of criteria established by an expert group in 1997. ${ }^{1}$ In some individuals, easy bruising, characteristic facial features, and thin skin with a readily visible venous pattern lead to the diagnosis. In most individuals, however, the diagnosis is not suspected (in the absence of a family history of the disorder) until arterial aneurysm and/or dissection, bowel perforation, or organ rupture occurs. Even in the presence of these complications, the identification of a mutation in COL3A1 confirms the diagnosis. ${ }^{2}$ Skin hyperextensibility, a hallmark of other common forms of Ehlers-Danlos syndrome, is rarely seen as a major feature in vEDS. ${ }^{3}$ The prevalence of vEDS has been estimated to be about 1 in 90,000 , but this estimate is likely to be low. ${ }^{4}$ We previously described our experience with 220 families with a total of 419 affected individuals in which we focused on the events that led to diagnosis, complications that characterized the group, and survival. ${ }^{5}$ We have now expanded this group to include 630 index individuals and 601 relatives with vEDS, identified the COL3A1 mutation in 572 families, characterized elements of the genotype/phenotype relationship, and widened our understanding of clinical presentations, survival that is mutation dependent, and elements that limit our ability to have full ascertainment. These data should, therefore, provide a more detailed means by which to counsel individuals and families and to demonstrate that mutation identification will be important in the design of future clinical studies.

\section{MATERIALS AND METHODS}

Study individuals

The 630 index individuals included 572 individuals in whom confirmation of a COL $3 A 1$ disease-causing mutation was present and 58 individuals whose cultured dermal fibroblasts, studied before genetics testing was available, synthesized abnormal type III procollagen molecules. The COL $3 A 1$ mutation or confirmed protein alteration from 611 of the index cases was identified in the Collagen Diagnostic Laboratory, University of Washington Department of Pathology, between 1976 and 2012, and 13 were identified in other diagnostic laboratories. In each of the latter group of families, the mutations were confirmed independently in an affected family member in the Collagen Diagnostic Laboratory. Based on the medical records that accompanied the diagnostic sample submitted for laboratory testing, we determined the reasons for the initial referral to a physician and assessed the medical history, family history, and physical findings, as available.

We used three criteria to designate 601 relatives of the index cases as having vEDS. Either a COL3A1 mutation or type III collagen protein defect was identified (321 relatives), the family history of the index individuals indicated obligate affected status, or an individual had had a major complication that was 
consistent with the diagnosis: arterial rupture, dissection, or aneurysm, bowel perforation, or organ rupture (280 relatives). Using this approach, the individuals (relatives) who did not have a mutation and had not experienced a major complication were omitted (Table 1).

On the basis of records that accompanied diagnostic samples of the 630 index cases and 321 relatives, we determined the number and type of medical complications, the ages at which they occurred, surgeries and outcomes, the cause of and age at death, reported birth defects, and complications of pregnancy. The age of ascertainment of the index individuals was the age at which we confirmed the diagnosis by testing. For affected relatives, the age at ascertainment was the age at which we identified them by genetic testing, their last known age, or their age at death as recorded in the family history. Limited medical histories were available for the remaining 280 relatives who were identified by a review of the family history of the index cases.

The age at ascertainment was known for 1,177 of the 1,231 affected individuals and ranged from 1 to 87 years of age (Table 1). Seventy percent of the index individuals (450 of 630) were ascertained because of a major complication; the remaining 180 index individuals were ascertained either because of a positive family history ( $23 \%$ of all index cases) or on the basis of physical examination (7\%). The primary features of those identified by physical examination alone were chronic easy bruising and striking distal joint hypermobility. Characteristic facial features, thin skin with visible veins, and/or an associated birth defect were noted in some individuals.

To standardize clinical data for a comparison among families and subjects, major complications were classified as "vascular," "gastrointestinal," or "organ." In some instances, it was the first

Table 1 Characteristics of vascular Ehlers-Danlos syndrome study population

\begin{tabular}{|c|c|c|c|}
\hline Characteristics & $\begin{array}{c}\text { All } \\
\text { subjects }\end{array}$ & $\begin{array}{c}\text { Male } \\
\text { subjects }\end{array}$ & $\begin{array}{l}\text { Female } \\
\text { subjects }\end{array}$ \\
\hline Number & 1,231 & 594 & $\begin{array}{c}633(+4 \text { gender } \\
\text { unknown) }\end{array}$ \\
\hline Index patients & 630 & 316 & 314 \\
\hline Relatives & 601 & 278 & $\begin{array}{c}319(+4 \text { gender } \\
\text { unknown) }\end{array}$ \\
\hline $\begin{array}{l}\text { Mean age at } \\
\text { ascertainment }\end{array}$ & 27.9 & - & - \\
\hline Index & $\begin{array}{l}27.4 \text { years } \\
(n=615)\end{array}$ & $\begin{array}{c}27.2 \\
(n=309)\end{array}$ & $\begin{array}{c}27.7 \\
(n=306)\end{array}$ \\
\hline Relatives & $\begin{array}{l}31.6 \text { years } \\
(n=562)\end{array}$ & $\begin{array}{c}29.6 \\
(n=261)\end{array}$ & $\begin{array}{c}33.7 \\
(n=301)\end{array}$ \\
\hline \multicolumn{4}{|c|}{ Family history of disease in index patients } \\
\hline Yes & $232(37 \%)$ & 105 & 127 \\
\hline No & $275(43 \%)$ & 144 & 131 \\
\hline Unknown & $125(20 \%)$ & 67 & 58 \\
\hline Age at first complication & 30.6 years & 29.7 & 31.6 \\
\hline $\begin{array}{l}\text { Number of subjects with } \\
\text { age at first complication } \\
\text { known }\end{array}$ & $n=670$ & $n=354$ & $n=316$ \\
\hline
\end{tabular}

of several complications that was included because one "event" could include numerous complication types, tissues, and locations in the course of a hospitalization.

We analyzed data about pregnancy outcome and report the outcomes of that study separately. ${ }^{6}$

\section{DNA sequence analysis and analysis of type III procollagen} In the majority of affected individuals, the diagnosis was confirmed by COL3A1 sequence analysis. Until 1999, the primary sequence analysis was performed in complementary DNA, and the mutation was confirmed in genomic DNA. Subsequently, sequence analysis used genomic DNA as the substrate and Big Dye chemistry, with resolution of fragments by automated capillary separation (amplification and sequencing primers are available on request). Along with mutations that disrupted splice sites, an analysis of RNA (complementary DNA) was performed to characterize the outcome(s) when dermal fibroblasts were available. For relatives, only the region of COL3A1 that contained the familial mutation was amplified and sequenced. For 58 affected individuals, the diagnosis was confirmed solely by biochemical analysis of type III procollagen and collagen in cultured dermal fibroblasts. ${ }^{5}$

\section{Statistical methods}

Survival was calculated with the Kaplan-Meier estimator for all individuals and stratified by sex and mutation type. Hazard ratios (HRs) that described associations between time to event and mutation type, mutation location, and amino-acid substitution were assessed using Cox regression. ${ }^{7}$ When we compared splice-site mutations with other types, we removed those that result in IVSX-1G>A from the "acceptor site" population because they are predicted to lead to frameshifts and mRNA instability when the first nucleotide of the exon is G. Estimated standard errors for all Cox regressions were "robust," i.e., they allowed for clustering (i.e., nonindependence) of time to event within families. ${ }^{8}$ Groups were compared using the robust score test, equivalent to the log-rank test, which allowed for clustering within families. A survival curve derived from the 2008 age-specific death rates in the Division of Vital Statistics of the Centers for Disease Control and Prevention (http://www. cdc.gov/nchs/products/vsus.htm\#appendices) was used for a comparison with our study population. The incidence rates for clubfoot, congenital hip dislocation, and amniotic bands were derived from the NCBI National Library of Medicine, (http:// www.ncbi.nlm.nih.gov/pubmedhealth/PMH0002208/, http:// www.ncbi.nlm.nih.gov/pubmedhealth/PMH0001966/, and http://www.ncbi.nlm.nih.gov/pubmedhealth/PMH0002546/). The incidence rate for limb deficiency was derived from the USCDC National Registry (http://www.cdc.gov/ncbddd/birthdefects/data.html). Each recorded national incidence rate was compared with the study population.

We used the calculated expected proportion of substitutions for glycine residues in the triple helical domain that would result in single amino-acid changes mutations that would lead to each substitution for glycine as described by Persikov et al. ${ }^{9}$ 


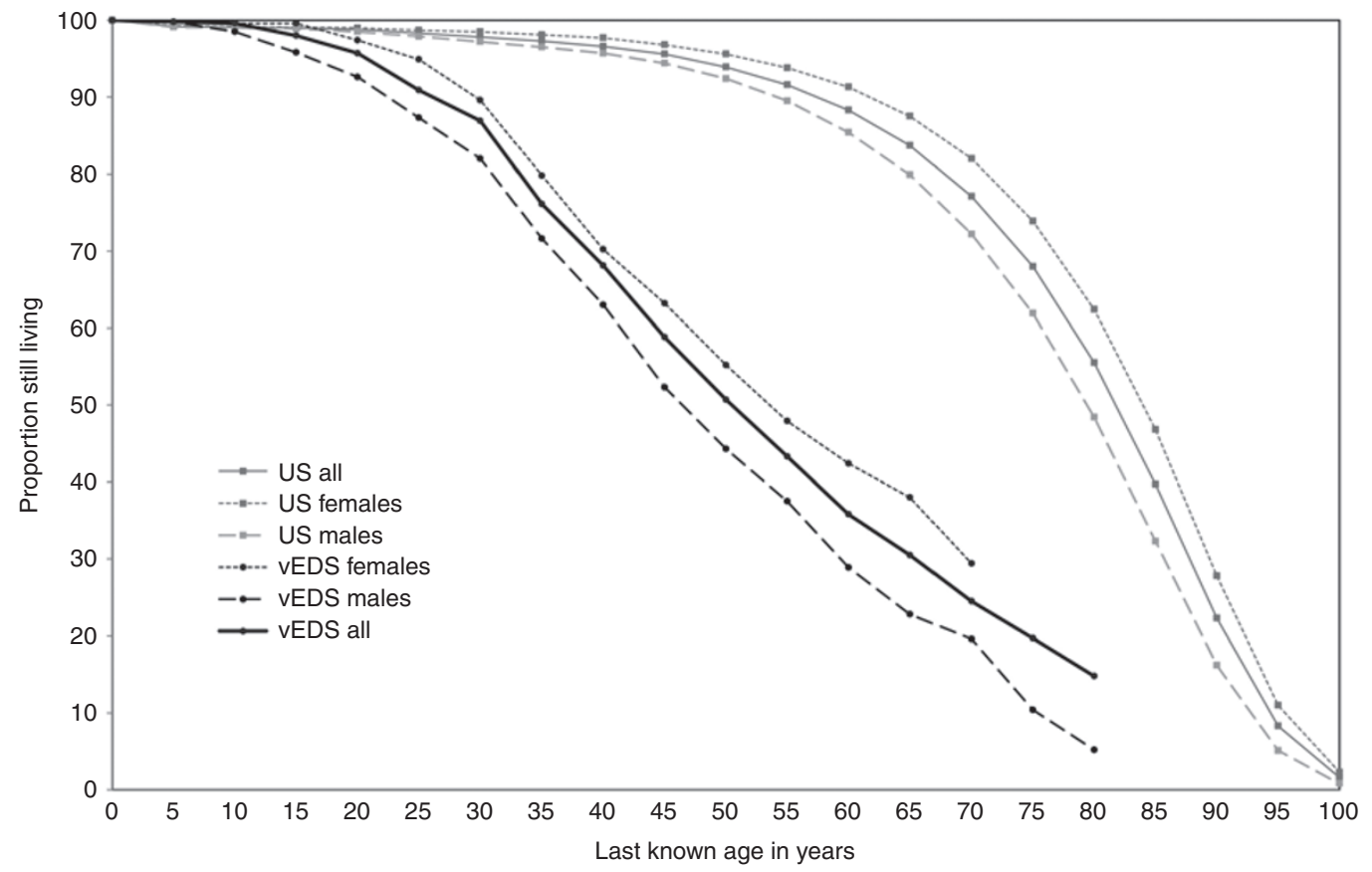

Figure 1 Kaplan-Meier survival curve comparing vascular Ehlers-Danlos syndrome (vEDS) study population to 2008 US population.

Comparisons of grouped populations were statistically calculated using the Microsoft Excel data analysis tool.

\section{RESULTS}

\section{Mutations}

We identified the underlying causative COL3A1 mutation in 572 of the index individuals (410 different mutations) (Supplementary Table S1 online). Among the families with mutations, 356 had alterations that led to substitutions for glycine in the repeated Gly-X-Y triple motif of the triple helical domain. Another 164 had a mutation that disrupted a splice donor site (144) or splice acceptor site (20). The remaining 52 had unique insertions, duplications, deletions, or insertions/ deletions. In 27 individuals, the described mutation led to loss of a stable mRNA transcript from one allele, which was a null mutation. Of the 410 unique mutation sites, 69 had more than one family with the same mutation. Among these, four sites (c.1662+1G $>$ A, IVS24+1G $>$ A (32), c.547G $>$ A, p.Gly183Ser, Gly16Ser in the triple helical domain (18), c.755G $>\mathrm{T}$, p.Gly252Val, c. 1347+1G >A, IVS20+1G>A (9), and Gly85Val in the triple helical domain (8)) accounted for $30 \%$ of recurrent mutations. There were 17 additional sites with more than 2 unrelated families with the same mutation.

Single-nucleotide substitutions in glycine codons (GGN) can give rise to codons for eight other amino acids and a single termination codon. The observed distribution of substitutions that resulted from single-nucleotide substitutions in glycine codons in the triple helical domain differed significantly $(P$ $<0.01 \chi^{2}$ test, in Excel) from that which had been predicted (Supplementary Table S2 online). Substitutions of glycine codons by those for small or neutral amino acids (alanine, serine, and cysteine) were less frequent than expected.

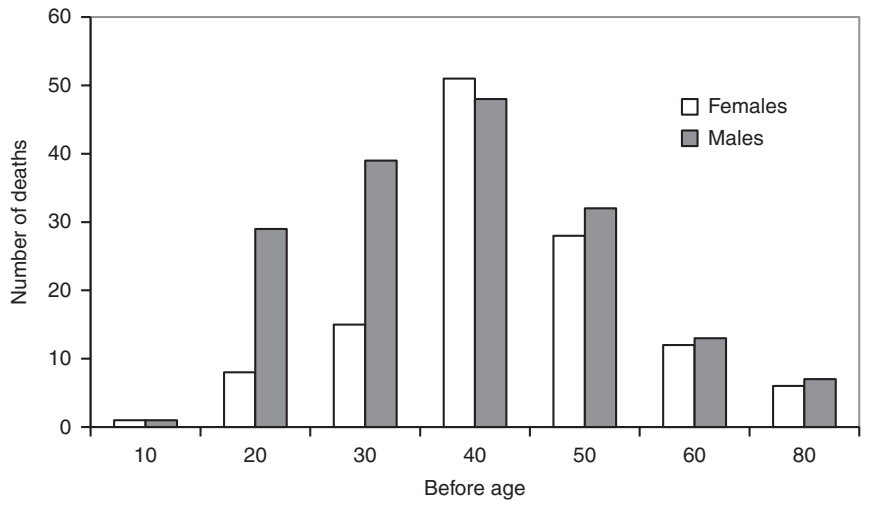

Figure 2 Number of deaths by decade in males and females of vascular Ehlers-Danlos syndrome study population.

\section{Survival}

The median survival for all individuals in the study was 51 years as measured by the Kaplan-Meier estimator. Median survival age for males was $46 \pm 1.77$ years $(95 \%$ confidence interval (CI): $42-51)$, and that for females was $54 \pm 2.46$ years (95\% CI: 49-63) $(P<0.0001$, log-rank test) (Figure 1). The gender difference appeared to stem from a relative excess of deaths in young males. Among males, $18 \%$ of deaths $(n=30)$, all as a consequence of vascular dissection or rupture, occurred before age 20 compared with $7 \%$ for females $(n=9)(P=$ $1.34 \times 10^{-5} \chi^{2}$ test). For individuals in whom the age of death was known, $41 \%$ of male deaths and $26 \%$ of female deaths were identified in the first three decades of life. The proportion of deaths in males and females is close to equal by age 40 , but the excess female deaths in this period does not appear to be the result of pregnancy-specific complications (Figure 2). ${ }^{6}$ 


\section{a}

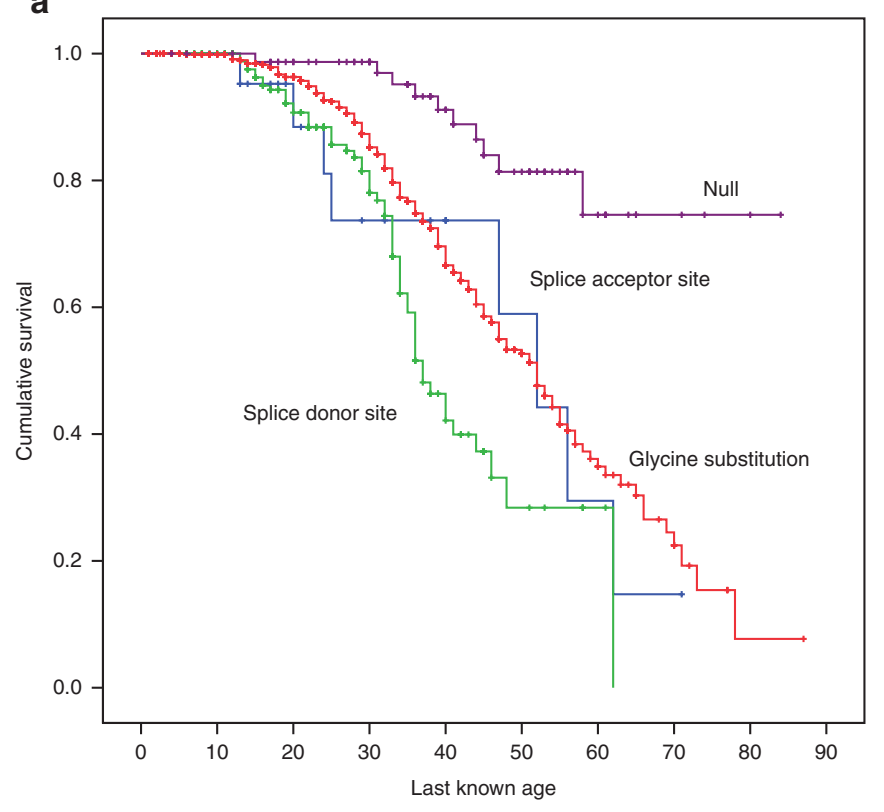

b

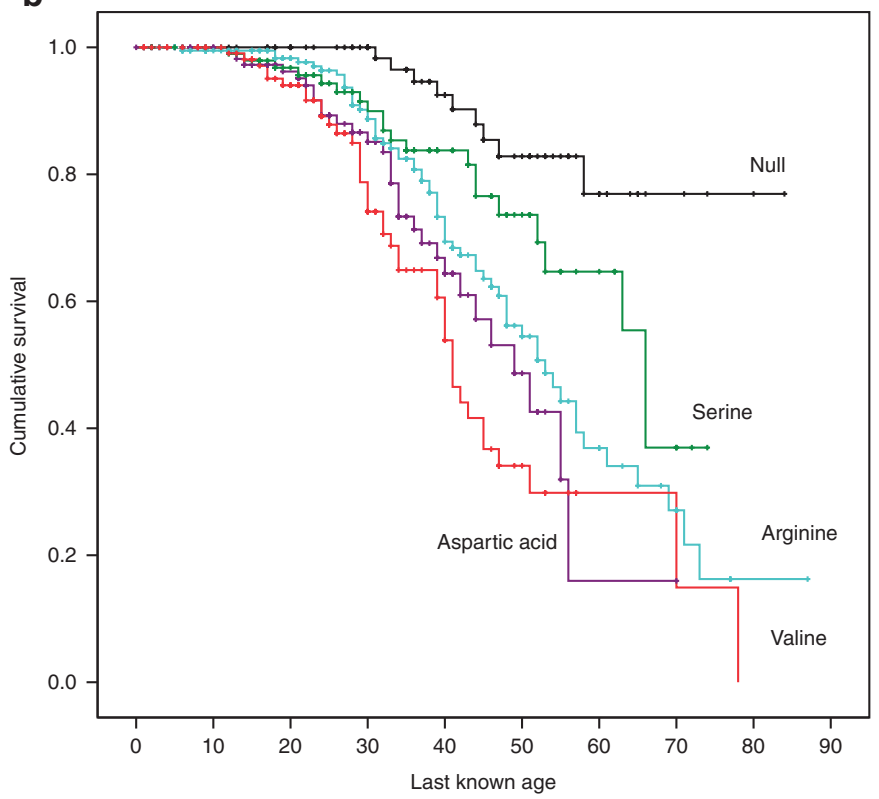

C

\begin{tabular}{|l|c|c|c|}
\hline Substitution & $\begin{array}{c}\text { Median } \\
\text { survival in } \\
\text { years }\end{array}$ & $\begin{array}{c}\text { Estimated } \\
\text { hazard } \\
\text { ratio }\end{array}$ & $95 \% \mathrm{Cl}$ \\
\hline Null & 43 & 5.54 & $(2.2,14.1)$ \\
\hline Alanine & (ref) & (ref) \\
\hline Cysteine & 49 & 5.30 & $(2.7,10.6)$ \\
\hline $\begin{array}{l}\text { Aspartic } \\
\text { acid }\end{array}$ & 34 & 5.66 & $(2.7,11.8)$ \\
\hline $\begin{array}{l}\text { Glutamic } \\
\text { acid }\end{array}$ & 52 & 4.01 & $(2.1,7.8)$ \\
\hline Arginine & 66 & 2.62 & $(1.2,5.5)$ \\
\hline Serine & 41 & 7.17 & $(3.7,13.8)$ \\
\hline Valine & & & \\
\hline
\end{tabular}

Figure 3 Kaplan-Meier survival curve of vascular EDS study population comparing mutation type (a) and glycine amino acid substitutions (b) to null mutations. Median survival and $95 \%$ confidence interval $(\mathrm{Cl})$ of subjects with glycine substitution types and hazard ratio as compared to null (c).

\section{Survival difference across mutation types}

Median survival rates for individuals with acceptor site mutations, which excluded the IVSX-1G>A alterations that result in mRNA instability and nulls, donor site mutations, and substitutions for glycine in the triple helical domain were 52, 37, and 51 years, respectively (Figure 3). The median survival in the null mutation group could not be estimated because of the small number of deaths. Compared with null mutations, all three mutation types had a higher hazard ( $\mathrm{HR}=5.4,(95 \% \mathrm{CI}$ : 2.3-13.0), 8.8 (95\% CI: 4.63-16.7), and 4.6 (95\% CI: 2.5-8.4), respectively), and there was a significant difference in hazard across all four groups $\left(P=1.4 \times 10^{-6}\right.$, log-rank test $)$. Although the survival of individuals with mutations that led to substitutions for glycine in the triple helical domain was significantly shorter than for those with null mutations $\left(P=4 \times 10^{-5}\right.$, log-rank test), among those with substitutions, the identity of the replacement amino acid played a role in survival (serine $>$ arginine/aspartic acid $>$ valine for those with sufficient mutations to rank) ( $P=0.03$, log-rank test) that was not influenced by gender (Figure 3 ).

\section{Survival difference across mutation locations}

With substitutions for glycine that result from mutations in COL1A1 and produce osteogenesis imperfecta, both the substituting residue and the site along the triple helical domain affect the phenotype. ${ }^{10}$ When we grouped substitutions for glycine in 100 residue sets along the triple helical domain of COL3A1, we found no difference in survival among locations $(P=0.35, \log$-rank test $)$. Adjusting this analysis for gender made no difference. 
Table 2 Anatomic location of vascular complications in the vascular Ehlers-Danlos syndrome study population

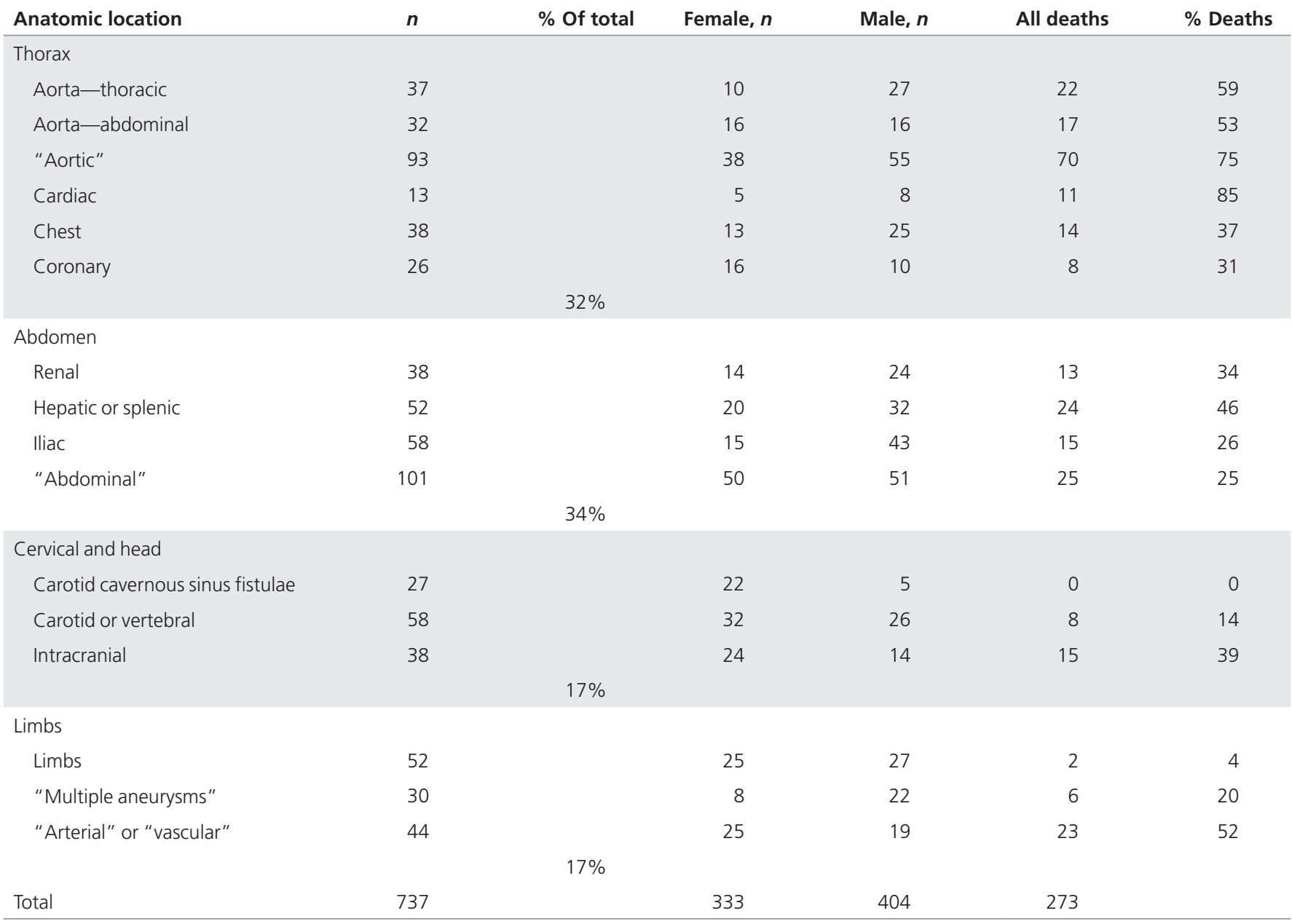

\section{Arterial complications}

We identified 737 arterial complications in 638 individuals. These findings included aneurysm, dissection, and rupture that typically occurred in large- and medium-sized vessels. The greatest number of events occurred in abdominal (34\%) and thoracic vessels (33\%), and then in head, neck, and extremities (Table 2).

\section{Aortic dissection/aneurysm or rupture}

Aortic aneurysm, dissection, or rupture was the leading single vascular complication (22\%) recorded and led to death in $68 \%$ of cases. The anatomic location of the aortic event was available in 162 instances: "aortic" $(n=88)$, "thoracic" $(n=14)$, "ascending," "arch," or "descending" $(n=23)$, "entire" $(n=5)$, and "abdominal" $(n=32)$. Males were identified with aortic events more often and at a younger age compared with females (males, $n=99$ and females, $n=63$ ). The mean age of aortic complication was 35.7 years (males, 32.6 and females, 41.0) ( $t$-test assuming unequal variance $P<0.01$ ). Aortic surgery was recorded in 32 instances, and in 39\% of the described cases, death ensued $(n=13)$. In 9 of the 13 instances $(69 \%)$, death occurred during emergency or exploratory surgery. The remaining four instances occurred during an aortic graft repair, mitral valve replacement, coronary bypass surgery, and endovascular surgery for carotid artery aneurysm (Supplementary Table S3 online).

\section{Spontaneous coronary artery dissections}

Coronary artery aneurysm, dissection, or rupture was described in 25 individuals (9 males and 16 females) (Supplementary Table S4 online). The mean age of the event was 30.8 years (25.0 for males and 33.5 for females). The presenting symptom for 17 of the 20 individuals (those with available data) was chest pain or symptoms described as typical of a "heart attack," including arm, substernal, jaw, or radiating chest pain. Only in rare instances had the pain been previously experienced $(n=$ 2: after exercise for 1 year; radiating jaw pain 2 weeks earlier in the immediate postpartum period). Sixteen of the 24 individuals survived the event, either without intervention (4 of 24) or with surgical repair (10 of 24) (10 of 24 unknown). Of those who died $(n=8)$, five died immediately without intervention and three died of complications either during or after surgery, each with evidence of a second extra-cardiac vessel complication. Confirmation of coronary dissection by angiography was 
recorded in nearly half of the cases (Supplementary Table S4 online).

\section{Cervical vascular complications}

Twenty seven carotid cavernous sinus fistulae were described ( 22 in females, 5 in males). The mean age of event was 30.9 years for both males and females. All individuals survived the event, with documentation of embolization repair in 17 instances. Seven of the 17 individuals recorded suffered arterial dissection along the course of the procedure $(n=5)$ or at the surgical entry site $(n=2)$.

Lethal cerebral vascular accidents without detailed description were reported in 15 of the 38 individuals who suffered "intracranial" events. The location of the dissection or rupture included "brain," "intracranial," and "cerebral." Cervical artery dissections (carotid or vertebral) were noted in 58 individuals ( 26 males, 32 females) and led to death in 8 individuals (Table 2).

\section{Gastrointestinal complications}

There were 181 bowel perforations described in this cohort; $65 \%(n=117)$ "colon" or "bowel" perforations; $29 \%(n=52)$ "sigmoid colon"; $4 \%(n=8)$ "small bowel"; and 2\% $(n=4)$ esophagus or stomach. The anatomic location and surgical details of perforations, beyond the fact that they occurred, were rarely recorded. For this reason, only descriptive data for "sigmoid" colon perforations were summarized. In 9 of the 52 individuals who experienced sigmoid perforation as the presenting complication, repair was associated with death. In each of those instances, unexpected hemorrhage or artery rupture was encountered at the time of repair. In the majority of instances of sigmoid rupture, the primary surgical approach was partial colectomy, creation of a colostomy, and reversal after several months $(n=25)$. In a much smaller number $(n=$ 3 ), the treatment consisted of primary repair (Supplementary Table S5 online).

\section{Congenital defects}

Four birth defects occurred with a significantly greater frequency than the currently estimated prevalence in the United States $(P<0.01, Z$-test). Clubfoot or unilateral or bilateral defect was recorded in $8 \%$ of study subjects. Congenital hip dislocation, limb deficiency, and amniotic band were recorded in roughly $1 \%$ of cases, which was well above the estimated US prevalence (Supplementary Table S6 online).

\section{Ascertainment of affected children}

We identified 295 individuals with vEDS less than 18 years of age; $60 \%(n=174)$ were tested in the presence of a positive family history. Of those $174,13 \%$ had experienced a major complication, and the mean age of testing was 8 years. Among individuals less than 18 years of age with a negative family history, the proportion ascertained on the basis of a major complication was greater (52\%), and the age of testing was older (mean of 11.5 years). In the absence of a positive family history or a major complication, the features most commonly identified as the reason for testing were easy bruising, thin skin, and joint hypermobility, in combination with clubfoot.

\section{DISCUSSION}

This study extends the work that was previously reported ${ }^{5}$ in that it contains the original data set and adds an additional 410 index cases and 402 relatives to bring the cohort to 1,231 individuals. The extended size of the cohort and our ability to identify mutations in most individuals has enabled us to develop a view of phenotype-genotype relationships that can help guide trials in the future and understand the effects of mutations in the clinical setting.

\section{Life span and mutation}

Individuals with vEDS and mutations in COL3A1 have, on average, a diminished life span. In the last decade and a half, we identified a set of index individuals who were heterozygous for mutations that led to loss of the stable mRNA from one COL3A1 allele (null mutations). ${ }^{11}$ These mutations introduce premature termination codons that lead to mRNA instability through the process of nonsense-mediated mRNA decay or, less frequently, a change in the sequence of the C-propeptide that interferes with chain association. These families are distinguished from those with other classes of mutation by a 10-15 year delay in onset of complications. The marked variation in expression in these families is consistent with the presence of genetic modifiers that we have not identified. ${ }^{11}$ At the time we completed our previous study, neither the number of mutations nor their diversity enabled us to define genotype-phenotype relationships.

We identified several different classes of mutations that included missense alterations (substitutions for glycine residues in the triple helical tripeptide Gly-X-Y repeat region), premature termination codons, and splice donor and splice acceptor site alterations. In the cohort of 1,231 cases (index individuals and their relatives), the median survival is 51 years. Families with "null" mutations that result in either mRNA instability or proo1(III) chain instability have the longest survival of all individuals with $C O L 3 A 1$ mutations. For individuals with missense mutations that result in substitutions for glycine in the triple helix, the HR is 4.6 (95\% CI: 2.5-8.4) compared with those with null mutations. The nature of the substituting amino acid has an effect on survival. Substitutions by serine results in an HR that is 2.6 times that of null mutations; by glutamic and aspartic acid, an HR that is five times greater than the null mutations; and by valine, an HR of 7:1. Donor site mutations that typically lead to exon skipping afford the highest HR (8.8), followed by the substitution for triple helical glycines (4.6), and acceptor site alterations (4.4) other than the $-1 \mathrm{G}>\mathrm{A}$ substitutions that result in null alleles. Acceptor site mutations are uncommon, and the outcomes are variable, and, in some instances, result in several mRNA products (see complete data in Supplementary Table S1 online). This stratification of effect on longevity has significant implications for the selection and assortment of individuals to arms in clinical trials. 


\section{Gender, longevity, and complications}

There was a 5-year difference between males and females in Kaplan-Meier survival curve. A significant part of the shift in survival among males was a higher death rate before age 20 , which was due to vascular rupture. The time from birth to 20 years accounted for $18 \%$ of deaths in males but for $7 \%$ of deaths in females. From age 20 on, the survival curves for men are women are parallel, and pregnancy did not appear to reduce predicted survival. ${ }^{6}$ Gender effects were seen in other ways: females accounted for $67 \%$ (16 of 25) of coronary artery dissections and for $80 \%$ of carotid cavernous sinus fistulae. Coronary artery dissection generally presented as a "heart attack" and comprised 3\% of all vascular complications. Strokes, initially considered relatively uncommon among people with vEDS, ${ }^{12}$ represented about $10 \%$ of all vascular complications and was often associated with carotid and vertebral artery dissection. ${ }^{5}$

\section{Surgical outcomes}

Arterial or bowel ruptures usually lead to decisions about surgical intervention. As screening for arterial enlargement becomes more common, the potential for improved outcome by surgical intervention remains a primary focus. In this study, the principal data collection followed unexpected events. Among those who were taken to surgery, records indicate that $70 \%$ survived. The descriptions of the surgery and associated complications were, however, scarce. Surgical outcome was worst for individuals who required emergency surgery for arterial events, and in this group there was a 70\% death rate. Surgical intervention in individuals who presented with carotid cavernous sinus fistulae, coronary artery dissection, and bowel rupture was usually successful. Even in these instances, suture dehiscence, bleeding from fragile tissues, infection, fistulae, adhesion, and repeat intervention were reported.

The present treatment for sigmoid colon rupture has become partial colectomy, colostomy, and reanastomosis (range 3 months-23 years). Only three instances of primary anastomosis came to our attention. Two of the three had postsurgical complications: abscess and abdominal bleeding in one instance; enterocutaneous fistulae in the other. In sigmoid colon repair, four of the nine deaths were documented to have resulted from rupture of nearby arteries. There were occasional reports of repeat perforation or complications at ostomy sites.

The extent of dissection and size of the vessel was related to the success of intervention but the unexpected tear, bleed, or rupture of nearby tissues, organs, and vessels all contributed to poor surgical course. Carotid cavernous sinus fistula repair by coil embolization was successful in all described cases (data were available for 13 of the 27 cases). Coronary artery dissection treatment by stent placement was usually successful.

Cautionary measures, when possible, with regard to handling of tissues and vessels during procedures may improve outcome. Records noted vessel tears at the entry site, along the path to destination during angiography, and during clamping or handling of friable vessels. Colon rupture during or after colonoscopy (1) or enema (2) was recorded.

\section{Early deaths and tests in children}

Death that occurred in the first two decades of life in our study population almost always resulted from spontaneous artery rupture or dissection. Arterial rupture, $60 \%$ of which involved the aorta, was responsible for all deaths in young males. It was striking that $53 \%(n=21)$ of teen deaths occurred in undiagnosed individuals without a family history of vEDS. We could not determine from the data we had whether there was preexisting aortic enlargement or dissection. The striking excess of deaths in young males, previously commented on by Barabas, ${ }^{13}$ raises the question of whether assessment of the arterial tree in the second decade in those who can be identified as a result of their positive family history could identify changes that would increase the risk for rupture or dissection.

Children with vEDS are most likely to be studied on the basis of a positive family history, chronic easy bruising, dramatic joint hyperextensibility, thin skin, and the presence of associated birth defects. Because the incidence of birth defects noted is likely under-represented, children with congenital dislocated hips, club feet, limb defects, and amniotic bands might be a population to screen by adding an extended family history as a genetic tool at the time the birth defect is identified.

Historically, genetic testing of children at risk for vEDS was considered "pre-symptomatic testing" for an adult-onset disorder. However, as our experience with the condition evolved, more children with vEDS were identified by complications at younger ages and the requests for testing of children increased. Nonetheless, the data in this study reveal that testing of children is not usually dictated by symptomatology. Of the 174 children with a positive family history of vEDS who were tested before age $18,87 \%$ had not experienced a major complication and the mean age of testing was 8 years. Parents of atrisk children do not wait until a complication arises (mean age 10.5 for the $13 \%$ who experienced a complication) or until the child reaches majority for a test to be performed but instead request that the test be performed to clarify the child's status and anticipate medical risks.

\section{Mutations in the population of affected individuals}

It has been difficult to measure the incidence or prevalence of vEDS in any population because it is difficult to come to the diagnosis in the absence of either the known complications or genetic tests that identify a $C O L 3 A 1$ mutation. The best population estimate comes from Sweden, where there are at least 100 known affected individuals in a population of 9 million, which is consistent with a prevalence of more than 1 in 90,000. ${ }^{4}$ At face value, 1,231 identified individuals in a population of more than 300 million people is a population frequency of a little more than 1 in 300,000. This disorder is not well recognized, and the diagnostic odyssey traveled before final diagnosis is often long. The mutation distribution that we identified differs significantly from the expected mutation pattern if there were no ascertainment bias. For example, almost half of all identified COL1A1 mutations 
that lead to osteogenesis imperfecta result in null mutations (Collagen Diagnostic Laboratory, University of Washington, unpublished data) compared with less than $5 \%$ of the mutations found in people with vEDS. This alone would augment the expected rate to more than 1 in 150,000, and correction for the expected number of mutations found in other classes would substantially augment it further. Mutations in COL1A1 account for more than $60 \%$ of mutations found in people with osteogenesis imperfecta. This disorder has an estimated frequency of about 1 in 10,000, so COL1A1 mutations alone should account for about 1 in 16,000. Given the similarity between the two genes, an estimated incidence of 1 in 25,000 for vEDS is probably not unreasonable. This means that unexplained long-term bruises, death before age 20 as a consequence of arterial rupture, aneurysms, dissection, or rupture in the vascular tree before age 45 , spontaneous (nondiverticular) bowel perforation, and uterine rupture and complex tears during delivery should all trigger consideration of this diagnosis. Because both surgical and medical advances lead to improved survival and quality of life for individuals with vEDS, the role of early identification can only gain importance.

\section{ACKNOWLEDGMENTS}

The work was supported, in part, by funding from the Freudmann Foundation for Inherited Disorders of Connective Tissue at the University of Washington and the Department of Pathology.

\section{DISCLOSURE}

The authors declare no conflict of interest.

\section{REFERENCES}

1. Beighton P, De Paepe A, Steinmann B, Tsipouras P, Wenstrup RJ. Ehlers-Danlos syndromes: revised nosology, Villefranche, 1997. Ehlers-Danlos National Foundation (USA) and Ehlers-Danlos Support Group (UK). Am J Med Genet 1998;77:31-37.

2. Superti-Furga A, Gugler E, Gitzelmann R, Steinmann B. Ehlers-Danlos syndrome type IV: a multi-exon deletion in one of the two COL3A1 alleles affecting structure, stability, and processing of type III procollagen. J Biol Chem 1988;263:6226-6232.

3. Steinmann B, Superti-Furga A, Royce PM. The Ehlers-Danlos syndrome. In: Steinmann B, Royce PM (eds). Connective Tissue and Its Heritable Disorders: Molecular, Genetic and Medical Aspects. Wiley-Liss: New York, 1993.

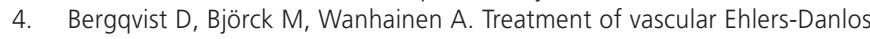
syndrome: a systematic review. Ann Surg 2013;258:257-261.

5. Pepin M, Schwarze U, Superti-Furga A, Byers PH. Clinical and genetic features of Ehlers-Danlos syndrome type IV, the vascular type. N Engl J Med 2000;342:673680.

6. Murray ML, Pepin M, Peterson S, Byers PH. Pregnancy-related deaths and complications in women with vascular Ehlers-Danlos syndrome. Genet Med 2014;16:874-880 (this issue).

7. Cox DR. Regression models and life-tables. J Roy Stat Soc: Ser B (Methodol.) 1972;34:187-220.

8. Thernau T. A Package for Survival Analysis in S. Vol R package version 2.36142012.

9. Persikov AV, Pillitteri RJ, Amin P, Schwarze U, Byers PH, Brodsky B. Stability related bias in residues replacing glycines within the collagen triple helix (GlyXaa-Yaa) in inherited connective tissue disorders. Hum Mutat 2004;24:330337.

10. Marini JC, Forlino A, Cabral WA, et al. Consortium for osteogenesis imperfecta mutations in the helical domain of type I collagen: regions rich in lethal mutations align with collagen binding sites for integrins and proteoglycans. Hum Mutat 2007;28:209-221.

11. Leistritz DF, Pepin MG, Schwarze U, Byers PH. COL3A1 haploinsufficiency results in a variety of Ehlers-Danlos syndrome type IV with delayed onset of complications and longer life expectancy. Genet Med 2011;13:717-722.

12. North KN, Whiteman DA, Pepin MG, Byers PH. Cerebrovascular complications in Ehlers-Danlos syndrome type IV. Ann Neurol 1995;38:960-964.

13. Barabas AP. Ehlers-Danlos syndrome type IV. N Engl J Med 2000;343:366; author reply 368. 